\title{
CDISC CDASH Exposure Route of Administration Terminology
}

National Cancer Institute

\section{Source}

National Cancer Institute. CDISC CDASH Exposure Route of Administration Terminology. NCI Thesaurus. Code C78425.

A terminology subset of the CDISC Study Data Tabulation Model (SDT M) Route codelist created for Clinical Data Acquisition Standards Harmonization (CDASH) Exposure Route of Administration terminology. 